IRA-International Journal of Technology \&

Engineering

ISSN 2455-4480; Vol.10, Issue 04

Pg. no. 55-61.

Institute of Research Advances

https://research-advances.org/IRAJTE.html

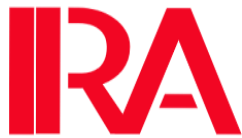

\title{
Brain Tumor Detection and Segmentation: A Survey
}

\author{
Kalifa Shantta ${ }^{1 \#}$ and Otman Basir ${ }^{2}$ \\ 1.2 Electrical and Computer Department, University of Waterloo, 200 University Ave W, Waterloo, \\ ON N2L 3G1, Canada.
}

\# corresponding author

Type of Work: Peer Reviewed.

DOl: http://dx.doi.org/10.21013/jte.v10.n4.p1

\section{How to cite this paper:}

Shantta, K., Basir, O. (2018). Brain Tumor Detection and Segmentation: A Survey. IRA-International Journal of Technology \& Engineering (ISSN 2455-4480), 10(4), 55-61.

doi:https://dx.doi.org/10.21013/jte.v10.n4.p1

(C) Institute of Research Advances.

\section{(cc)) BY-No}

This work is licensed under a Creative Commons Attribution-Non Commercial 4.0 International License subject to a proper citation to the publication source of the work.

Disclaimer: The scholarly papers as reviewed and published by the Institute of Research Advances (IRA) are the views and opinions of their respective authors and are not the views or opinions of the IRA. The IRA disclaims of any harm or loss caused due to the published content to any party.

Institute of Research Advances is an institutional publisher member of Publishers International Linking Association Inc. (PILA-CrossRef), USA. The institute is an institutional signatory to the Budapest Open Access Initiative. Hungary advocating the open-access of scientific and scholarly knowledge. The Institute is a registered content provider under Open Access Initiative Protocol for Metadata Harvesting (OAI-PMH)

The journal is indexed \& included in WorldCat Discovery Service (USA), CrossRef Metadata Search (USA), WorldCat (USA), OCLC (USA), Open J-Gate (India), EZB (Germany) Scilit (Switzerland), Airiti (China), Bielefeld Academic Search Engine (BASE) of Bielefeld University, Germany, PKP Index of Simon Fraser University, Canada. 


\begin{abstract}
Even with the enormous progress in medical technology, brain tumor detection is still an extremely tedious and complex task for the physicians. The early and accurate detection of brain tumors enables effective and efficient therapy and thus can result in increased survival rates. Automatic detection and classification of brain tumors have the potential to achieve efficiency and a higher degree of predictable accuracy. However, it is well established that the accuracy performance of automatic detection and classification techniques varies from technique to technique, and tends to be image modality dependent. This paper reviews the state-of-the-art detection techniques and highlights their pros and cons.
\end{abstract}

Keywords: Brain Tumor, Automatic Detection, Accurate Detection, Classification Accuracy.

\title{
Introduction
}

The cancer cells grow and form the mass of tissue known as a tumor. In normal circumstances, body cells die and are exchanged for new ones. To a certain degree, the presence of cancerous and other tumors disrupts this phase. Although the body does not require them, tumor cells multiply, and such cells do not die, as healthy cells do. As this process continues, the cancer keeps on growing as additional cells are added to the mass. The expression "glioma" is used to describe a rapidly spreading primary brain tumor. Gliomas originate from glial tissue, which assists and sustains the cells that transmit messages from the brain to different parts of the body. Brain tumors can be benign or malignant (cancerous). Non-cancers tumors are benign growths in the body that cannot invade neighboring tissue. They can be completely removed and are unlikely to reappear. Benign brain tumors do not spread to adjacent tissue; however, they can cause significant pain, lasting brain damage, and death. Malignant brain tumors have no distinct limits. They grow quickly, creating increasing pressure within the brain, and can also diffuse throughout the brain or spinal cord beyond their point of origin. It is extremely rare for malignant brain tumors to spread outside the brain.

\section{Brain Tumor Detection}

As per the survey has done the highest death rate in the world is due to a brain tumor. Symptoms include changes in the hormones, blood clots, weakness, uncontrolled walking, muddled speech, mood swings, loss in vision, etc. The location of the tumor defines its type, and its proper diagnosis can save the life of the patient[1]. Benign tumors are non-cancerous growths in the body that cannot invade neighboring tissue. They can be completely removed and are unlikely to reappear. Benign brain tumors do not spread to adjacent tissue; however, they can cause significant pain, lasting brain damage, and death. Malignant brain tumors have no distinct limits. They grow quickly, creating increasing pressure within the brain, and can also diffuse throughout the brain or spinal cord beyond their point of origin. It is extremely rare for a malignant brain tumor to spread outside the brain.

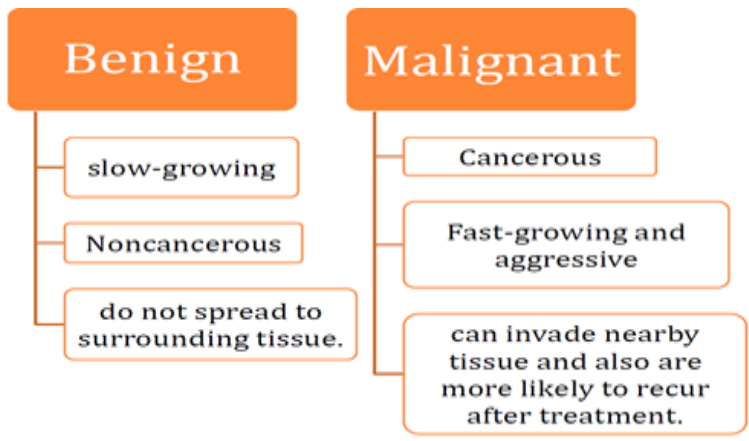

Fig. 1 Types of brain tumor

Figure.1 shows the tumor types and their brief characteristics. Techniques like MRI or CT scan gives the complete structure of a brain tumor as it directs into the intracranial cavity producing a clear image of the tumor. MRI scan scans by using strong magnetic fields and high radio frequencies to provide detailed information of soft tissues. Compounded Tomography scan scans by sending X-ray beams. Steps involved in the detection of brain tumors are pre-processing of an image, feature extraction, segmentation, and post-processing of the image. 


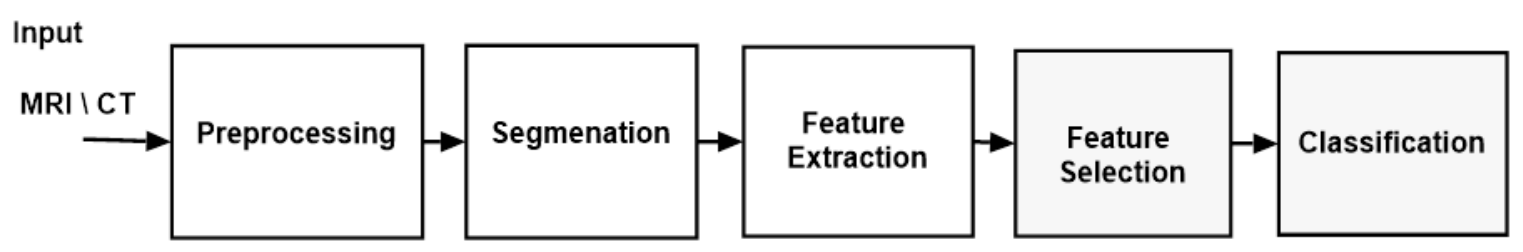

Fig. 2. Stages in brain tumor detection systems

Figure 2 demonstrates the required stages for any automatic brain tumor detection.

\section{Image Preprocessing}

Image pre-processing is a significant aspect of any image-based application. Pre-processing stage is required for the following reasons:

1. Pre-processing prepares the images for higher-level processing such as segmentation and feature extraction.

2. Remove the marks or labels such as name, date, and other details (film artifacts) in the image that can affect the classification task.

3. Image quality needs to be enhanced.

4. Removal of any types of noise in the image.

\section{Image Segmentation}

The aim of image segmentation is to divider a medical image into different regions and to extract the area of interest. In particular, it is used for separating components from the remainder of the image so that they can be observed or recognized as objects.

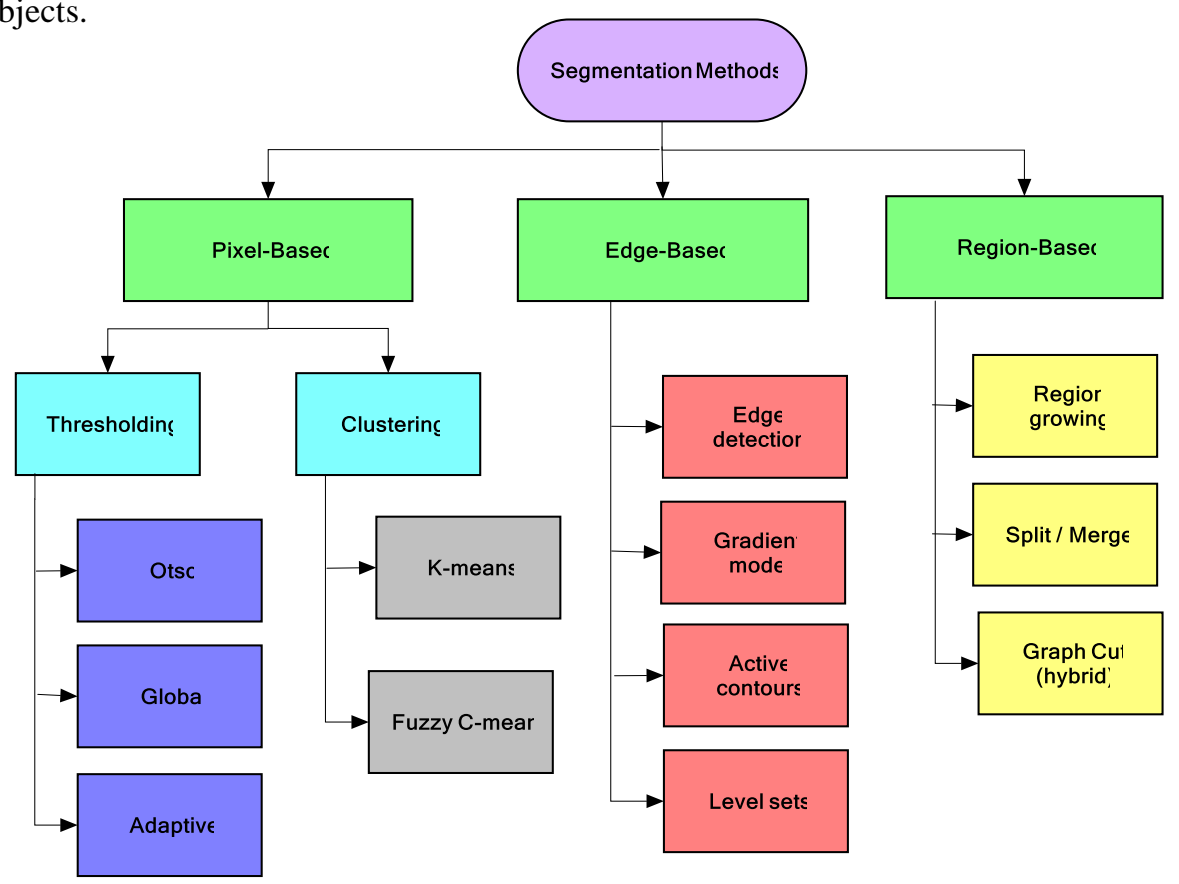

Fig. 3. Segmentation categories

Alireza et al. [2] divided image segmentation into three groups, as illustrated in Figure 3: pixel methods, regionbased methods, and edge-based methods. Alireza et al. [2] and Yu Jin [3] also published general surveys of image segmentation. Some reviews targeted the segmentation of MRI images in particular [4]-[ 5]. Zhang et al. [6] and Clarke et al. [7] provided direct comparisons of diverse approaches for segmenting MRI images. 


\section{Feature Extraction and Selection}

Nassiri et al. [8] defined the objective of feature extraction as the minimization of the original data set based on the computing of specific characteristics or attributes that classify and identify different input patterns. The dimensionality reduction is one of the goals from the feature extraction stage, which accurately identifies interesting components of an image as a compact feature vector. This technique is useful for application with large images, for which feature representation must be reduced to enable the fast completion of tasks such as image matching and retrieval.

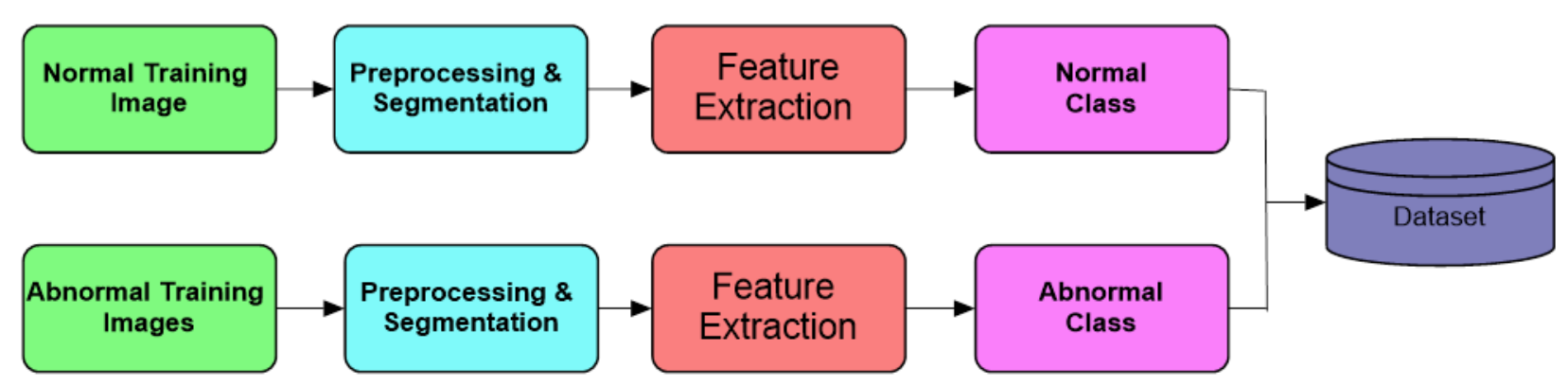

Fig.4. Block diagram of feature extraction stage

The block diagram of the feature extraction phase is demonstrated in Figure 4. Ziedan et al. [9] demonstrated that the best-known feature extraction approaches are local binary pattern (LBP), grey-level co-occurrence matrix (GLCM), Canny edge detection, and a bag of words (BoW). Numerous research studies have been undertaken to compare the features of available extraction techniques [10]-[11].

The objective of feature selection is the elimination of unrelated and unnecessary features from input records to choose a subgroup of related features for the construction of robust classifiers. This step will probably increase the building speed and precision of the final classifier. From a theoretical perspective, it can be claimed that ideal feature selection for supervised learning tasks requires a comprehensive search of all probable feature subsections. Nevertheless, for a considerable number of features or models, conducting a complete examination of all features for the creation of an optimal feature set is impractical. For this reason, a supervised learning algorithm is employed for analyzing an appropriate approximation of the best set of features for a specific classifier rather than for determining an optimal set.

\section{Classification Algorithms}

In the areas of classification and machine learning, researchers have expanded techniques and computer applications to accomplish a particular objective. Their work indicates the development of particular learning techniques for enhancing the achievement of a standard based on the use of model data or prior events [12]. During supervised learning, the approval of the training is dependent on patterns that carry output labels.

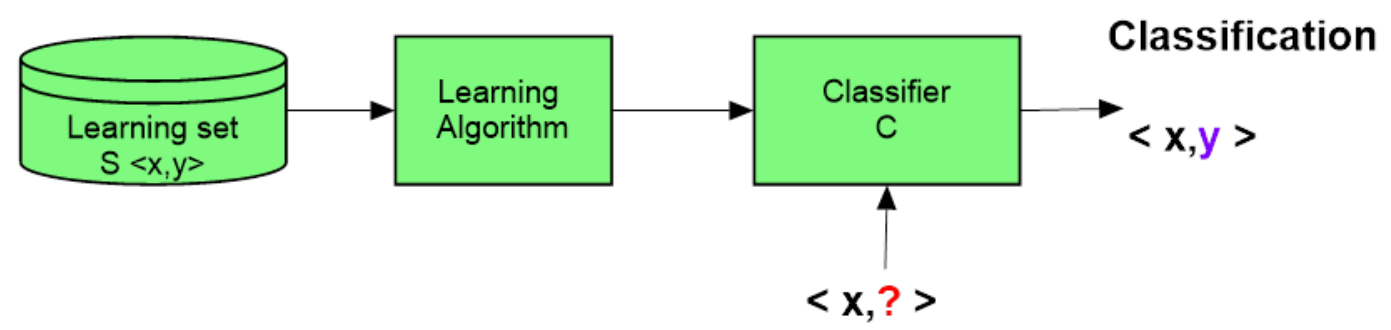

Fig. 5. Supervised learning model

Figure 5 shows the supervised learning model. When the output values identify the various classes to which the samples relate, the training assignment is called "classification." The classifier factors have described the training data consistently because they have been collected from the model data. The training data set to be fed to the classification algorithm for constructing the classifier consists of $\mathrm{N}$ data points that can be formally denoted as $\left\{x_{i}\right.$, 
$\left.y_{i}\right\}^{\mathrm{N}} \mathrm{n}=1$, with $x_{i} € R^{d}$ being an input example of dimension $d$ and $y_{i} €\{-1,+1\}$, the corresponding class label for a two-class classification task.

\section{Literature Survey}

Adel Kermi et al [13] proposed automatic brain cancer segmentation procedure in three dimensional-magnetic resonance imaging utilize similarity analyses of brain and standard group. Preprocessing of photograph is done to remove noise. The FBB technique is effective and unsupervised. Using the FBB method, the detection of tumors is done automatically. A geodesic level set based $3 \mathrm{~d}$ deformable model is applied to detect the boundaries of the tumor, in any case of its form and volume. The average calculation time of detecting and segmenting tumors is around five minutes. The accuracy and sensitivity obtained were $38.04 \%$ and 89.01 respectively.

V. Anitha, S. Murugavalli [14] presented the technique of clear and systematic examination using an algorithm. information of lesions is specific by segmentation of MRI, which is based on anatomical structures and potential abnormal tissue data. The $\mathrm{K}$ means techniques are applied to achieve successful segmentation and classification by a two-tier approach. The feature extraction obtained after applying the discrete wavelet transform and used to learning the neural network's self-organizing map and the outcome filter factors are then learned by the KNN neighbor and the testing procedure is similarly done in double phases. It has better performance than traditional classification methods and the experiment indicates enhanced performance. Two-tier classification segmentation systems organize the regular and irregular MRI efficiently. MATLAB R2013a platform is used to apply the algorithm. sensitivity and specificity expressions are applied to a statistical measure of this two-level classifier method. The indicated outcome marks the superiority over SVM based classification technique and also indicates that it can be incorporated in medical imaging application for image classification and also in CAD. They achieved $85 \%$ in the accuracy factor, and $100 \%$ in the sensitivity.

Parveen and Iitpalsingh [15], proposed data mining techniques for the classification of magnetic resonance imaging photos. Classification is accomplished in four stages: preprocessing, partition, attributes extraction, and classification. In the first stage, improvement and skull stripping is undertaken to increase speed and accuracy. A fuzzy C-means gathering technique is applied during the segmentation stage. The extraction of magnetic resonance imaging photos features is performed using a grey level matrix. The final stage applies SVM for classifying the images. The final results of this study exhibited a high degree of accuracy and efficiency with respect to MRI image classification.

Daniele Ravi et al. [16] propose a new dimensionality reduction and processing method to achieve a detailed structural map for into operative margin definition. Due to manifold embedding and inconsistent results in other dimensionality reduction techniques, tissue classification is hindered. While this method does the same work in two phases - first The tissue classification is done after $\mathrm{T}$ distributed stochastic neighbor process flowed by semantic segmentation method using Semantic Texton Forest. The suggested system can support in realization of cancer development. The actual time nature of the methods can perfect clinical precision, offering extra knowledge that can as well minimize the likelihood of faulty re-sectioning of healthy tissue. The great quality and precision of the gained tumor maps can be obtained by applying a proper establish way shared with a classifier that considers only the spectral worth of every pattern nevertheless similarly their spatial context. The accuracy and sensitivity achieved were 81.90 and 80.91 respectively.

Lamia Salemi et al. [17] presented a convivial algorithm for Glioblastoma modelization. The extracted tumor region is done by applied by rapid spreading matching based global pixel wise-data. The new model uses an algorithm related to cellular automata and a speedy marching technique to assess the cancer evolution during the time. This method has an optimized runtime of less than 0.5 seconds for every image and does not need long training. Glioblastoma presents different gray level potency compared to safe cells. This information is used to slice the brain image into two regions. Regions with Glioblastoma is then matched with the estimated model based on intensity levels. The proposed system extracts the tumors in real-time which is then used for growth estimation using CRMM.

Mukambika P. S., Uma Rani K. [18] suggested phases that the MRI Image was processed in 4 phases: Preprocessing, Segmentation, patterns extraction, and pattern recognition stages. In the pre-processing stage, the skull is removed from the MRI image by applied a double thresholding procedure The proposed research introduces the comparative learning of dual methods applied for cancer recognition of MRI pictures. The first method is established by utilizing the non-parametric deformable models with an active contour to section the brain cancer 
from the magnetic resonance imaging brain pictures. The second method is the $\mathrm{K}$-means segmentation technique. A decision making is achieved in dual phases next to segmentation: DWT applied for feature extraction and "Gray Level Co-occurrence Matrix". finally, SVM utilized in the classification stage. Dataset of MRI brain tumor images includes T2 weighted 17 benign and 24 malignant tumor images of different patients. SVM with Level Set and KMeans segmentation classifies the image into normal brain, benign or Malignant tumor with $94.12 \%$ and $82.35 \%$ accuracy respectively The level set produces the best outcome compared to K-means segmentation.

K. Sudharani, Dr. T. C. Sarma, Dr. K. Satay Rasad [19] Proposed Methodology includes methods like Histogram, Re-sampling, K-NN Algorithm, Distance Matrix. First, Histogram provides the whole amount of the stated amount of pixels spread in a specific image. Re-sampling re-size picture to $629 \times 839$ for appropriate geometrical representation. Brain tumor is classified and identified by applied the KNN algorithm after tuning the K parameter. The distance is calculated by applied the Manhattan metric to classify. Labview was used to implement the algorithm. The data set contains 48 images were used to test the algorithm. The classification score for all tested images is around $95 \%$.

Rasel Ahmed, et al. [20] proposed method which includes stages like image pre-processing, segmentation, attributes extraction, SVM classification, and tumor stage classification using Artificial Neural Network(ANN). In preprocessing adjusted, adaptive threshold and histogram imaging was applied using both weiner 2 and median2 filter. Segmentation was applied by The TKFCM algorithm that is the mixing of the K-means and Fuzzy c-means with little adjustment. Feature extraction is done in two orders. In First order statistic features and Second-order region property based statistic features are derived. Then SVM classifies brain MRI pictures into ordinary or cancer brain. The brain tumor stage is classified by the ANN classifier. The dataset contains 39 images represent benign and malignant tumors. There are 3 images normal and 9 benign. The malignant images different grades. The proposed method achieved $97.44 \%$.

Ketan Machhale, et al[21]hey introduce an intellectual system that classifies MRI brain images to ordinary and malignant. Four phases were applied in this proposed system. Pre-processing stage followed by feature extraction, and finally the classification phase. Different classification techniques applied such as support vector machine and KNN to distinguish 50 images. They conclude that overall accuracy $98 \%$ obtained.

\section{Conclusion}

The tumor examination is a difficult and precise efforts, certainty and accuracy are consistently considerable attention. Thus a detailed methodology that focuses on the novel outline for expanding additional powerful image cancer segmentation as well detection approaches is much desired. In the paper, a partial survey of contemporary proposed methods for the designation of MR brain images is done. The accuracy, sensitivity, specificity, as well as their real-time applications, was applied to validate the performance of the utilized algorithms. Detecting brain tumors is a complex and sensitive task, so preciseness and reliability will similarly show an important part of the chosen method.

\section{References}

[1]. Chandana, S. R., Movva, S., Arora, M., \& Singh, T. (2008). Primary brain tumors in adults. American family physician, 77(10), 1423.

[2]. Norouzi, A., Rahim, M. S. M., Altameem, A., Saba, T., Rad, A. E., Rehman, A., \& Uddin, M. (2014). Medical image segmentation methods, algorithms, and applications. IETE Technical Review, 31(3), 199-213.

[3]. Zhang, Yu Jin. (2001).A survey on evaluation methods for image segmentation. Pattern recognition 1335-1346.

[4]. Wells, William M., et al. "Adaptive segmentation of MRI data." IEEE transactions on medical imaging 15.4 (1996): 429-442.

[5]. Sinha, A., Pratik, R., \& Panchal, M. (2014). MRI IMAGE SEGMENTATION-A REVIEW.

[6]. Zhang, Y., Brady, M., \& Smith, S. (2001). Segmentation of brain MR images through a hidden Markov random field model and the expectation-maximization algorithm. IEEE transactions on medical imaging, 20(1), 45-57.

[7]. Clarke, L. P., et al. "MRII segmentation: methods and applications." Magnetic resonance imaging 13.3 (1995): 343 368.

[8]. Nassiri, M. J., Vafaei, A., \& Monadjemi, A. (2007). Texture feature extraction using Slant-Hadamard transform. International Journal of Applied Science, Engineering and Technology, 3, 4.

[9]. Ziedan, R. H., Mead, M. A., \& Eltawel, G. S. (2016). Selecting the Appropriate Feature Extraction Techniques for Automatic Medical Images Classification. International Journal, 1. 
[10]. Li, J., Tseng, K. K., Zu Yi Hsieh, C. W. Y., \& Huang, H. N. (2014). Staining pattern classification of antinuclear autoantibodies based on block segmentation in indirect immunofluorescence images. PloS one, 9(12).

[11]. Medjahed, S. A. (2015). A comparative study of feature extraction methods in images classification. International journal of image, graphics and signal processing, 7(3), 16.

[12].Luts, J., Laudadio, T., Idema, A. J., Simonetti, A. W., Heerschap, A., Vandermeulen, D., ... \& Van Huffel, S. (2009). Nosologic imaging of the brain: segmentation and classification using MRI and MRSI. NMR in Biomedicine: An International Journal Devoted to the Development and Application of Magnetic Resonance In vivo, 22(4), 374-390.

[13].Kermi, A., Andjouh, K., \& Zidane, F. (2018). Fully automated brain tumour segmentation system in 3D-MRI using symmetry analysis of brain and level sets. IET Image Processing, 12(11), 1964-1971..

[14]. Anitha, V., \& Murugavalli, S. (2016). Brain tumour classification using two-tier classifier with adaptive segmentation technique. IET computer vision, 10(1), 9-17..

[15].Singh, A. (2015, February). Detection of brain tumor in MRI images, using combination of fuzzy c-means and SVM. In 2015 2nd International Conference on Signal Processing and Integrated Networks (SPIN) (pp. 98-102). IEEE.

[16].Ravì, D., Fabelo, H., Callic, G. M., \& Yang, G. Z. (2017). Manifold embedding and semantic segmentation for intraoperative guidance with hyperspectral brain imaging. IEEE transactions on medical imaging, 36(9), 1845-1857.

[17].Sallemi, L., Njeh, I., \& Lehericy, S. (2015). Towards a computer aided prognosis for brain glioblastomas tumor growth estimation. IEEE transactions on nanobioscience, 14(7), 727-733.

[18].Mukambika, P. S., \& Uma Rani, K. (2017). Segmentation and classification of MRI brain tumor. Int. Res. J. Eng. Technol.(IRJET), 4(07), 683-688.

[19].Sudharani, K., Sarma, T. C., \& Rasad, K. S. (2015, December). Intelligent Brain Tumor lesion classification and identification from MRI images using k-NN technique. In 2015 International Conference on Control, Instrumentation, Communication and Computational Technologies (ICCICCT) (pp. 777-780). IEEE.

[20].Ahmmed, R., Swakshar, A. S., Hossain, M. F., \& Rafiq, M. A. (2017, February). Classification of tumors and it stages in brain MRI using support vector machine and artificial neural network. In 2017 International Conference on Electrical, Computer and Communication Engineering (ECCE) (pp. 229-234). IEEE.

[21].Machhale, K., Nandpuru, H. B., Kapur, V., \& Kosta, L. (2015, May). MRI brain cancer classification using hybrid classifier (SVM-KNN). In 2015 International Conference on Industrial Instrumentation and Control (ICIC) (pp. 60-65). IEEE. 